in this respect, and might frequently be seen waiting at the pump in in the yard until some one came for water, when he would sit down quietly under a copious shower, stretching out his neck and ruffling his feathers up to allow the water to reach the skin. They do not like any exposure, and always endeavour to get out of the sun. In the wild state they seldom leave the scrubs, and certainly never do so in the heat of the day unless hard pressed; but on the whole they are remarkally hardy, and bear confinement well. In February last (1875) I purchased four fine young birds about 6 months old, which were obtained from some settlers in the Herbert-river district; these also I forwarded to England during the same month.

\title{
7. Description d'un nouveau Cerf tacheté du pays d'Ussuri méridional, Cervus dybowskii. Par L. Taczanowski, C.M.Z.S.
}

[Received December 17, 1875.]

En 1868, le Capitaine Przewalski a observé pour la première fois des cerfs tachetés dans ce pays, et dit dans son ouvrage imprimé en russe en 1870, que ces animaux abondent dans les grands forêts des côtes de la mer du Japon et de la région des affluents de l'Ussuri, en indiquant la rivière Tina pour limite septentrionale de la distribution de ce ruminant, de sorte qu'il n'en trouve point dans la région centrale du cours de l'Ussuri. M. Przewalski l'a nommé $C$. axis, Erxl. (?), et dit ensuite qu'il y a aussi dans ce pays un second cerf tacheté d'une taille intermédiaire entre le précédent et le cerf commun; et que sa peau d'été ne diffère en rien de celle de son $C$. axis, mais en hiver elle est d'un gris foncé, surtout au dos, où le poil est presque noir avec des taches blanchâtres à peine distinctes. Il a vu plusieurs fois des individus de cette espèce, sans pouvoir s'en procurer.

Le Dr. Dybowski vient de me fournir cinq peaux de ce cerf, parmi lesquelles il y a quatre mâles de différents âges (deux qui ont atteint le développement entier, un jeune à dagues simples, un d'âge intermédiaire) et une femelle. Ces cerfs me paraissent appartenir à cette dernière forme, celle plus forte du Cap. Przewalski, et tous ces exemplaires ont leur robe d'hiver. L'espèce paraît être inédite ; je propose donc de le nommer $C$. dybowskii, en l'honneur de mon ami, zoologiste plein de mérite, dont le séjour de dix ans dans la Sibérie orientale a sensiblement augmenté les connaissances de la faune de ce pays, si curieux et si insuffisamment exploré en faits d'histoire naturelle.

Ce cerf est d'une taille plus forte que celle du daim (C. dama) et beaucoup plus petite que celle du cerf commun.

Les bois sont élevés et minces, moins penchés que ceux du C.elaphus, et moins recourbés sur les côtés, à trois andouillers simples, dont le basilaire et le suivaut sont dirigés en avant un peu obliquement sur les côtés et légèrement courbés, et le troisième dirigé vers le milieu et très peu en arrière ; ces embranchements sont médiocres, et pres- 
que égaux entre eux; le prolongement du tronc principal est plus long que les branches précédentes, vertical, légèrement courbé vers le milieu. Le premier andouiller basal prend naissance beaucoup plus haut que celui du cerf commun (l'axe de cette branche est à 4 centim. de la couronne), le deuxième est plus éloigné du précédent que du troisième (19 et 17 centim.). Sur la face supérieure de la partie du tronc comprise entre les deux premiers andouillers se trouve une assez forte

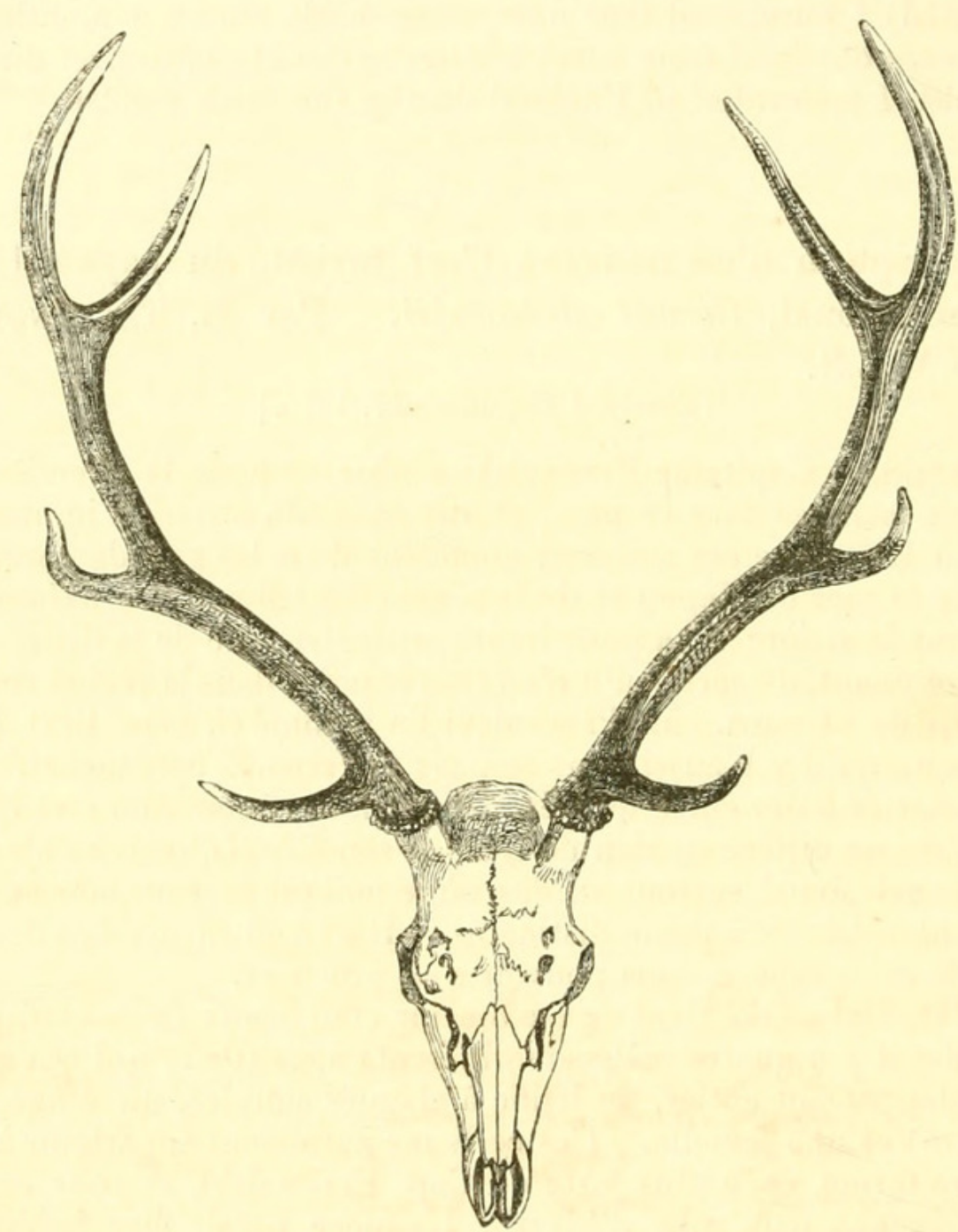

Skull and horns of Cervus dybowskii.

carène, et cette partie est considérablement plus haute que large ; l'anneau basal est étroit. La rugosité surtout dans la moitié basale est assez forte. Les appendices osseux servant de base aux bois sont très-élevés (5 centim.).

Le nez est nu; les fosses lacrymales profondes. La crinière est abondante au cou et entre les épaules, et se prolonge d'une manière distincte jusqu'à la naissance de la queue; tout le cou est garni de poils longs. La queue est beaucoup plus courte que dans le C. axis, garnie de longs poils, qui forment une touffe dépassant l'extrémité caudale de 22 centim. 
La couleur générale des animaux adultes en pelage d'hiver ressemble à celle d'un chevreuil (C. capreolus) d'hiver, c'est-à-dire qu'elle est formée d'un semis d'une multitude de petites stries fauves sur un fond gris brunâtre; chaque poil est gris, rembruni vers le haut avec un large anneau fauve à une certaine distance du sommet, qui est noirâtre. Sur ce fond à couleur de chevreuil, il y a sur la partie postérieure du corps des taches blanchâtres, peu distinctes. Le fauve roussâtre prédomine sur les côtés du cou, tandis qu'en dessous le fond principal est brunâtre, varié de fauve. La crinière est rousse sur la nuque, ensuite elle devient brune variée de roussâtre ; elle est distincte par une nuance beaucoup plus foncée dans toute la longueur du dos. Le museau est gris, piqueté de nombreuses stries fauves blanchâtres, cette couleur passe graduellement au roux sur le front; il y a une tache blanche, pure, cordiforme sur le devant du menton. La face dorsale des oreilles est roussâtre; l'interne est blanche ainsi que le bordexterne de la face dorsale dans sa moitié inférieure. La queue est plus ou moins rousse en dessus avec un mélange d'un petit nombre de poils noirs, et blanche en dessous. Il y a aussi un espace blanc au pourtour des fesses, couvert par une grande tache caractéristique noire. Le ventre est gris brunâtre presque uniforme avec un peu de blanchâtre au milieu et du blanc pur dans la partie postérieure. Les jambes sont fauves roussâtres avec une large raie brune le long de la face antérieure, et une tache blanche arrondie sur le côté externe au-dessous du talon dans les jambes postérieures.

Le jeune mâle à dague simple et un autre à ce qu'il paraît de deux ou trois ans, sont en général plus roux sur un fond brun plus foncé ; le roux prend un ton ferrugineux bien intense le long de la crinière dans toute la longueur du corps. Les taches blanchâtres sont beaucoup plus prononcées, mais elle paraissent être plus restreintes. La couleur roussâtre des jambes est plus vive, avec la raie dorsale brune plus foncée.

\section{Dimensions :}

centim

Hauteur des bois................. 58

Distance entre les bois à la base $\ldots \ldots \ldots \ldots \ldots . \ldots 9 . \ldots$

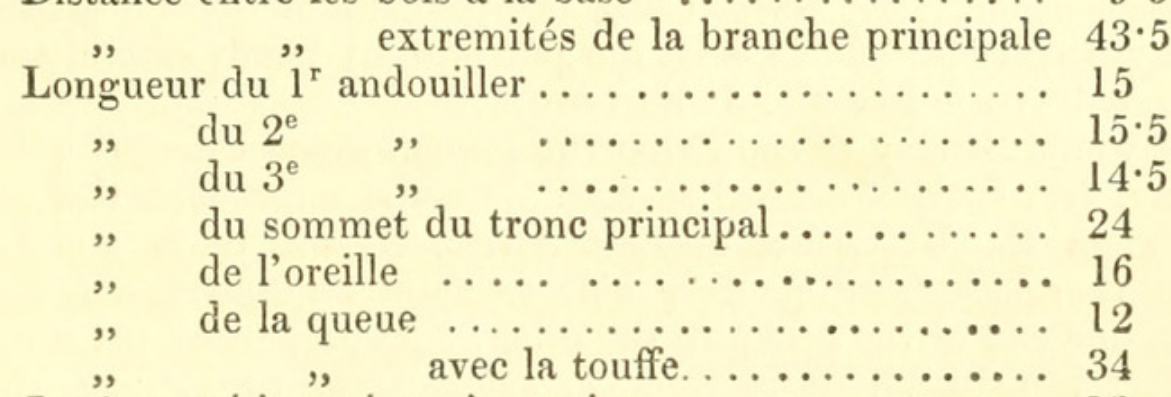

Jambe antérieure jusqu'au poing .......... 38

Tarse ...................... 48 


\section{$2 \mathrm{BHL}$ Biodiversity Heritage Library}

Taczanowski, Władysław. 1876. "7. Description d'un nouveau Cerf tacheté du pays d'Ussuri méridional, Cervus dybowskii." Proceedings of the Zoological Society of London 1876, 123-125.

https://doi.org/10.1111/j.1096-3642.1876.tb02548.x.

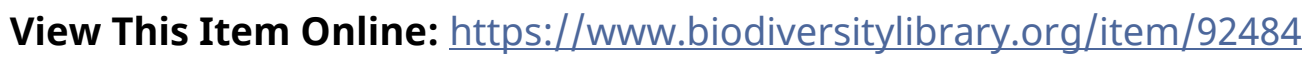

DOI: https://doi.org/10.1111/j.1096-3642.1876.tb02548.x

Permalink: https://www.biodiversitylibrary.org/partpdf/73570

\section{Holding Institution}

Smithsonian Libraries

\section{Sponsored by}

Smithsonian

\section{Copyright \& Reuse}

Copyright Status: Public domain. The BHL considers that this work is no longer under copyright protection.

This document was created from content at the Biodiversity Heritage Library, the world's largest open access digital library for biodiversity literature and archives. Visit BHL at https://www.biodiversitylibrary.org. 